\title{
Proper motion determination of open clusters based on the UCAC2 catalogue ${ }^{\star}$
}

\author{
W. S. Dias ${ }^{1,3}$, M. Assafin ${ }^{2}$, V. Flório ${ }^{1}$, B. S. Alessi, and V. Líbero ${ }^{1}$ \\ 1 Instituto de Física de São Carlos, Universidade de São Paulo, Caixa Postal 369, São Carlos 13560-970, SP, Brazil \\ e-mail: wilton@univap.br \\ 2 Observatório do Valongo, UFRJ, Ladeira Pedro Antonio 43, 20080-090 Rio de Janeiro, RJ, Brazil \\ 3 IP\&D, UNIVAP, Av. S. Hifumi 2911, São José dos Campos, 12244-000, SP, Brazil
}

Received 21 January 2005 / Accepted 16 September 2005

\section{ABSTRACT}

We present the kinematics of hundreds of open clusters, based on the UCAC2 Catalogue positions and proper motions. Membership probabilities were obtained for the stars in the cluster fields by applying a statistical method uses stellar proper motions. All open clusters with known distance were investigated, and for 75 clusters this is the first determination of the mean proper motion. The results, including the DSS images of the cluster's fields with the kinematic members marked, are incorporated in the Open Clusters Catalogue supported on line by our group.

Key words. Galaxy: open clusters and associations: general

\section{Introduction}

Recent results obtained from open cluster data have improved knowledge of the structure, dynamics, and evolution of the Galaxy and solar neighborhood.

The metallicity of open clusters with different ages and located at various Galactocentric radii allows the radial abundance gradient to be obtained, a fundamental constraint on investigating the evolution of the Galaxy (Chen et al. 2003). Radial velocities and metallicities can be determined by photometry and spectroscopy of cluster member stars. Together with other young objects like HII regions, open clusters are also suitable for tracing the spiral arms (Russeil 2003). Their spatial coordinates, ages, and motions permitted direct determination of both the speed of the spiral pattern and of the location of the corrotation radius (Dias \& Lépine 2005). For almost all of these investigations, correct discrimination between cluster member and field stars is crucial for accurate determining of the cluster's parameters, both the kinematical and especially the astrophysical ones.

Individual stellar proper motions in a few stellar astrometric catalogues display high internal precision in scales on the order of some arc minutes in the sky. This has proved useful in estimating the mean proper motion of open clusters. One advantage is that with such precise catalogues, it is now possible to make estimates for membership probabilities in a purely

* Tables 2 to 359 are only available in electronic form at the CDS via anonymous ftp to cdsarc.u-strasbg.fr (130.79.128.5) or via

http://cdsweb.u-strasbg.fr/cgi-bin/qcat?J/A+A/446/949 kinematic fashion and for a much larger number of stars. Until recently, such estimates were only possible with laborious procedures that almost always involved just a few of those stars with known and adequate photometric and spectrographic data. The availability of the Tycho 2 Catalogue (Høg et al. 2000) enabled our group to determine the astrometric membership of stars up to the 12th magnitude (Dias et al. 2001, 2002, hereafter DLA). The recently published UCAC2 Catalogue (Zacharias et al. 2004) provides high-precision proper motions for an even greater number of stars up to magnitude 16. Representing the most precise, complete, and up-to-date stellar census of proper motions, the UCAC2 allows statistically meaningful determinations of cluster membership for stars in the fields of hundreds of open clusters.

To determine the mean proper motion of star clusters and to select astrometric members based on the UCAC2 Catalogue data are the objectives of this work. In the next section, we describe the data used. In Sect. 3, we give the details of the procedures and of the method adopted for determining the membership and mean proper motion of the clusters. Results are presented and discussed in Sect. 4, while in the last section, we summarize the main results and give some concluding remarks.

\section{Data used}

From our database (The New Catalogue of Optically Visible Open Clusters and Candidates) ${ }^{1}$ (Dias et al. 2002, hereafter DAML02 Catalogue), we selected all clusters with known distances. We used version 2.0 of the DAML02 Catalogue, in

\footnotetext{
1 Available at http://www. astro.iag.usp.br/“wilton/
} 
which 740 clusters have determined distances (about $43 \%$ of the whole sample).

For extended clusters, the field contamination (typically thousands stars) does not allow useful member selection, while on the other hand, the number of UCAC2 stars in the region is not sufficient to apply the statistical analysis for some very small clusters. That is why we opted to discard $53 \mathrm{ob}-$ jects. Another 125 clusters cannot be investigated because they are out of the northern limit in declination of the UCAC2 Catalogue. The final list of open clusters investigated consists of 630 objects.

We searched for UCAC2 Catalogue stars in the sky area of the selected clusters, using the central coordinates and the apparent diameters taken from the DAML02 Catalogue. To include virtually all possible members of the studied clusters, we opted to use a region in the sky about 2 arcmin bigger than the area covered by the cluster. However, this is not a strict rule due to the individual needs of enlarging or reducing to avoid contamination of intruding field stars. The areas actually used for each cluster are presented in Table 1 of the astrometric results available in electronic form at the CDS. Extractions of the UCAC2 data have been performed using the VizieR tool at http://vizier.u-strasbg.fr/ viz-bin/VizieR?-source=I/ 289/out.

The UCAC2 Catalogue presents data for 48330571 sources covering the sky from $-90^{\circ}$ to $+40^{\circ}$ declination. Some areas go up to $+52^{\circ}$. The precision of the positions are 15 to 70 mas, depending on magnitude, with estimated systematic errors of 10 mas or below. Proper motions are derived by using over 140 ground- and space-based catalogues, including Hipparcos/Tycho (ESA 1997) and the AC2000.2 (Urban et al. 1998). The UCAC2 catalogue presents proper motions in the Hipparcos system with nominal errors of about 1 to $3 \mathrm{mas} / \mathrm{yr}$ for stars up to the 12 th magnitude and about 4 to $7 \mathrm{mas} / \mathrm{yr}$ for fainter stars up to the 16th magnitude. The systematic errors of the proper motions are below 0.5 to $1.0 \mathrm{mas} / \mathrm{yr}$ per coordinate (see details in Zacharias et al. 2004). The UCAC2 Catalogue thus represents the most complete and precise all sky stellar census of proper motions up to the 16th magnitude to date. This gives 4 more magnitudes and about 20 times more stars than using the Tycho-2 Catalogue and that without loss of accuracy, thus encouraging a new investigation with statistics at least as sound as in other similar, previous works (DLA).

\section{Mean proper motion and astrometric membership determination}

The segregation of cluster members in a sample of field stars in which an open cluster is present, should ideally be made using multicolor photometry, radial velocity, and proper motion data. On the other hand, practice shows that the close agreement between individual stellar proper motions and the cluster mean proper motion can be used with the appropriate statistical methods to determine cluster membership with good results. This technique was applied with relative success in a recent study of discovered clusters by our group (Alessi et al. 2003), and e.g. confirmed by the comparison with photometric selection in Clariá et al. (2003).

In the present study, the step procedures to determine a cluster's mean proper motion and identify astrometric members were as follows:

1) Considering one population: following the idea of Vasilevskis \& Rach (1957), we used all stars in the field and a Gaussian fit to estimate the average and standard deviation of the proper motions in each coordinate. As suggested by Zhao et al. (1982), stars with proper motions that are different from the mean by more than three standard deviations were discarded;

2) Considering two populations: the method consisted in fitting the observed distribution of proper motions with two overlapping normal bivariate frequency functions: an elliptical one for the field stars and a circular one for the cluster's. This is essentially the statistical method of Sanders (1971). However, to weight the stellar proper motions with different errors, we adopted the method of Zhao \& He (1990).

The set of nonlinear equations is not readily solvable, so an iterative procedure was adopted (see details in Sanders 1971). However, the initial values of mean proper motion in each coordinate are given in the first step, limiting the automatic search and allowing a faster and dependable convergence (Dias 2000).

Membership segregation was checked iteratively by plotting the distribution of membership probabilities and the vector proper motion diagram (VPD), thereby distinguishing those stars considered as members $(P \geq 51 \%)$ according to kinematical criteria exclusively.

In Table 1 we present the parameters provided by the method of Zhao \& He, with the mean proper motions of the clusters ( $\mu_{\alpha} \cos \delta, \mu_{\delta}$, error) and the number of cluster members. Are also given the results of the two-dimensional Gaussian fit to the population of field stars and the number of field stars, the orientation angle of the minor axis of elliptical field star proper motion distribution, and the radius (centered in the coordinates of the cluster) used to extract the stars from the UCAC2 catalogue. With the frequency function parameters, we could determine the individual membership probability of each cluster star. Tables 2 to 429 list the stars in the limits of each cluster that was sought, with the membership probabilities calculated by our method, derived from the proper motions. One part of the table for some stars in the region of the open cluster NGC 1662 is shown as an example, and all the tables are available in electronic form at the CDS and at the site of the DAML02 Catalogue.

\section{Discussion of the results}

Besides the unpublished results of mean absolute proper motions for 75 clusters, we could investigate the other 353 clusters and also determine their stellar memberships and mean absolute proper motions.

Our results cannot be compared directly to those in the literature previous to the Hipparcos mission, since they follow a 
Table 1. Example of the results of the proper motion analysis for open clusters. The meaning of the symbols are as follows: $N_{c}$ is the number of cluster stars; $N_{\mathrm{f}}$ is the number of field stars; $\mu_{\alpha} \cos \delta$ and $\mu_{\delta}$ are the proper motion components in mas/yr; err is the error of cluster' proper motions; $\sigma \mu_{\alpha} \cos \delta$ and $\sigma \mu_{\delta}$ are the dispersions of the components of the field stars' proper motions; $\theta$ is the orientation angle of the minor axis of elliptical field star proper motion distribution; $\mathrm{R}$ is the radius (centered in the coordinates of the cluster) used to extract the stars from the UCAC2 catalogue, in arcmin.

\begin{tabular}{lcccc|crrrrrr}
\hline \hline & \multicolumn{3}{c|}{ Cluster } & \multicolumn{1}{c}{ Field } \\
Region & $\mu_{\alpha} \cos \delta$ & \multicolumn{1}{c}{$\mu_{\delta}$} & err & \multicolumn{1}{c}{$N_{c}$} & $\mu_{\alpha} \cos \delta$ & \multicolumn{1}{c}{$\mu_{\delta}$} & $\sigma \mu_{\alpha} \cos \delta$ & $\sigma \mu_{\delta}$ & \multicolumn{1}{c}{$N_{\mathrm{f}}$} & \multicolumn{1}{c}{$\theta$} & $R$ \\
\hline Berkeley 21 & -0.6 & -6.2 & 0.5 & 32 & 2.9 & -7.4 & 5.5 & 10.4 & 50 & 84.641 & 5 \\
Bica 2 & -1.4 & -1.3 & 0.3 & 86 & 0.3 & 0.4 & 8.8 & 4.9 & 114 & 256.699 & 8 \\
Dias 4 & -4.9 & -4.1 & 0.8 & 19 & -8.9 & -6.8 & 9.2 & 8.8 & 29 & 160.100 & 3 \\
NGC 1662 & -1.4 & -2.4 & 0.3 & 55 & 1.9 & -7.7 & 7.1 & 7.2 & 104 & 63.388 & 15 \\
NGC 2304 & -3.8 & -6.9 & 0.7 & 20 & -2.1 & -1.3 & 14.5 & 15.3 & 25 & 343.979 & 3 \\
NGC 2422 & -7.1 & 0.7 & 0.3 & 170 & -6.3 & 8.7 & 1.5 & 5.5 & 565 & 269.535 & 13 \\
NGC 2849 & -7.3 & 4.5 & 1.0 & 12 & -13.1 & 4.9 & 12.1 & 10.8 & 38 & 271.357 & 4 \\
NGC 6216 & -2.1 & -6.9 & 0.8 & 15 & -2.9 & -5.2 & 16.5 & 14.8 & 55 & 296.313 & 3 \\
NGC 6318 & -0.4 & -2.4 & 0.4 & 45 & -1.7 & -3.4 & 11.8 & 11.8 & 97 & 45.795 & 4 \\
\hline
\end{tabular}

Table 2. Example of the UCAC2 stars analyzed in the searched limits of each cluster, with the membership probabilities calculated by our method. We present 10 lines of the last table, corresponding to stars of the region of the cluster NGC 1662. The UCAC2 data are exactly those extract from VIZIER (see comments in the text). $\sigma$ denotes the standard deviation. $P$ is the estimated membership probability (in percent).

\begin{tabular}{|c|c|c|c|c|c|c|c|c|c|c|}
\hline $\begin{array}{l}\text { 2MASS } \\
\text { id. }\end{array}$ & $\begin{array}{l}\alpha(\mathrm{J} 2000) \\
\text { hh mm ss }\end{array}$ & $\begin{array}{c}\delta(\mathrm{J} 2000) \\
\circ, \quad, \quad\end{array}$ & $\begin{array}{c}\sigma_{\alpha} \\
(\mathrm{mas})\end{array}$ & $\begin{array}{c}\sigma_{\delta} \\
(\mathrm{mas})\end{array}$ & magUCAC2 & $\begin{array}{l}\mu_{\alpha} \cos \delta \\
(\mathrm{mas} / \mathrm{yr})\end{array}$ & $\begin{array}{c}\mu_{\delta} \\
\text { (mas/yr) }\end{array}$ & $\begin{array}{c}\sigma_{\mu \alpha} \cos \delta \\
(\mathrm{mas} / \mathrm{yr})\end{array}$ & $\begin{array}{c}\sigma_{\mu \delta} \\
(\mathrm{mas} / \mathrm{yr})\end{array}$ & $\begin{array}{l}P \\
\%\end{array}$ \\
\hline 968277182 & 44827.873 & 105545.71 & 14 & 10 & 10.24 & -1.0 & -3.9 & 2.3 & 1.0 & 80 \\
\hline 968277178 & 44824.621 & 105544.29 & 15 & 30 & 15.85 & -2.8 & -8.0 & 5.4 & 5.5 & 13 \\
\hline 968277216 & 44830.590 & 105607.17 & 21 & 15 & 15.21 & -6.5 & -13.8 & 5.4 & 5.4 & 0 \\
\hline 968277154 & 44827.298 & 105514.68 & 15 & 17 & 11.04 & 4.1 & -3.4 & 1.6 & 1.6 & 12 \\
\hline 968277147 & 44827.843 & 105508.41 & 11 & 13 & 9.70 & -2.9 & -0.5 & 1.3 & 1.3 & 86 \\
\hline 968277150 & 44824.939 & 105509.12 & 10 & 12 & 9.51 & -1.8 & -1.6 & 1.1 & 1.1 & 88 \\
\hline 968277256 & 44834.491 & 105644.11 & 8 & 10 & 9.06 & -2.1 & -1.9 & 1.1 & 1.1 & 88 \\
\hline 968277157 & 44834.050 & 105516.93 & 31 & 15 & 15.64 & -6.0 & -0.8 & 5.5 & 5.4 & 50 \\
\hline 968277317 & 44832.083 & 105759.03 & 1 & 1 & 8.67 & -0.9 & -0.7 & 1.1 & 1.0 & 86 \\
\hline 968277233 & 44836.250 & 105620.74 & 72 & 22 & 15.01 & 6.8 & -13.9 & 6.1 & 5.4 & 0 \\
\hline
\end{tabular}

different system. However, they can be compared to those published by Baumgardt et al. (2000); Beshenov \& Loktin (2004); Kharchenko et al. (2003); and by our group, compiled in the DAML02 Catalogue. Even so, these authors used different data, methods, and criteria to select the members and, consequently, different samples of the stars to determine the mean proper motion of the clusters.

Baumgardt et al. (2000) determined mean proper motions and parallaxes for 205 open clusters from their member stars found in the Hipparcos Catalogue. To separate the cluster members from the field stars, the authors combined the Hipparcos data with information provided by ground-based studies: photometry and spectroscopy, proper motions, radial velocities, parallaxes, and angular distances from the cluster centers. Stars were divided into three categories (members, possible members, and non-members) according to how they fulfilled the membership criteria.

Kharchenko et al. (2003) obtained results of fundamental parameters and mean proper motions from membership determinations for 401 clusters. They used individual stellar data from ASCC-2.5 all-sky Catalogue (All-Sky Combined Catalog of Astrometric Data for 2.5 Million Stars; Gulyaev \& Nesterov 1992). For cluster membership, the stars ought to satisfy the photometric criterion of being within $1 \sigma$ of the color index
$E(B-V)$ to the left of the Zero Age Main Sequence in the Color Magnitude Diagram. Cluster membership probabilities of $61 \%$, $14 \%$, and $1 \%$ or less were assigned to stars with proper motions within, respectively 1,2 , or $3 \sigma$ or more from the cluster's mean proper motion. The cluster mean proper motion was computed based on the stars with $P \geq 61 \%$.

Beshenov \& Loktin (2004) investigated open clusters from their "Homogeneous catalogue of open clusters parameters" (Loktin et al. 2001) and determined proper motions for $390 \mathrm{ob}-$ jects. The authors used both kinematic (stellar proper motion) and photometric criteria to extract cluster members, and the final membership determination was computed by adopting a modified Sanders method (Loktin 2001). Then, only the stars with high membership probability $(P \geq 65 \%)$ were used to determine the proper motion of the cluster.

Our group determined the mean proper motion of hundreds of open clusters and astrometric membership of stars up to the 12th magnitude using the Tycho2 data and the statistical method of Sanders (1971). We also used a different database - the UCAC2 instead of Tycho2 - as well as the method by Zhao \& He (1990), which represents a slight improvement over the previous method of Sanders (1971), because individual errors in the stellar proper motions are now taken into account. Otherwise, the mean absolute proper motions and membership 

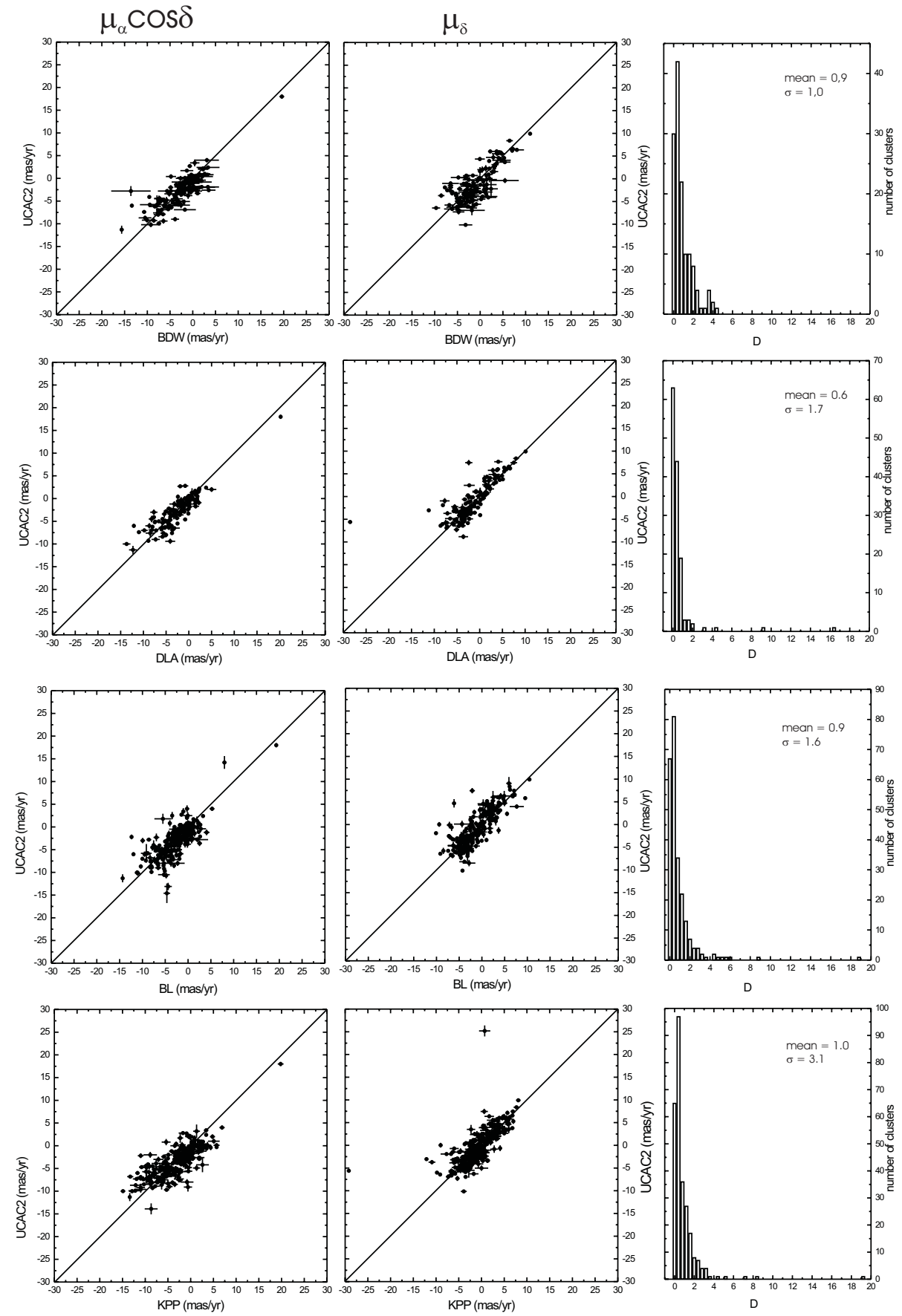

Fig. 1. Comparison of our results for mean proper motions with those provided by Baumgardt et al. (2000); Dias et al. (2001, 2002); Beshenov \& Loktin (2004), Kharchenko et al. (2003) (BDW, DLA, BL, $\mathrm{KPP}$, respectively); The lines of $45^{\circ}$ are the loci of equal proper motion. The right panels present the distribution of the proper motion differences for each comparison, taking into account the right ascension and declination components weighted by the formal errors (quantity $D$, see text); they indicate that no statistical distinction exits between the compared proper motion distributions within the estimated errors.

determinations in both investigations were performed in much the same fashion, and they both considered essentially the same fundamental hypotheses and computational tools.

One can see from Fig. 1 that the mean proper motions of the open clusters from all these studies are reproduced well and are not dominated by errors. To check the quality of our own mean proper motions, we compared the differences in the right ascension and the declination components for each cluster, weighted by the formal errors computing the following quantity (D):

$$
\begin{array}{r}
D=\frac{\left(\mu_{\alpha} \cos \delta_{\mathrm{UCAC} 2}-\mu_{\alpha} \cos \delta_{\text {literature }}\right)^{2}}{\left(\sigma_{\mu \alpha} \cos \delta_{\mathrm{UCAC} 2}\right)^{2}+\left(\sigma_{\mu \alpha} \cos \delta_{\text {literature }}\right)^{2}} \\
+\frac{\left(\mu_{\delta \mathrm{UCAC} 2}-\mu_{\delta \text { literature }}\right)^{2}}{\left(\sigma_{\mu \delta \mathrm{UCAC} 2}\right)^{2}+\left(\sigma_{\mu \delta \text { literature }}\right)^{2}} .
\end{array}
$$

In Fig. 1 (right panels) we present the distribution of the results (D) for each comparison. In all cases, almost all values (D) lie below 2.5 to 3 . Thus, we can confidently state there is no statistical distinction between the compared proper motion distributions within the estimated errors.

Essentially, the same typical rms proper motion found in literature is reproduced here for these common clusters, but with a much larger number of identified cluster member stars. The total number of stars studied in the fields is about 150000 , of which about 42000 were considered astrometric members. This is a considerable improvement over our previous works (DLA), as about 13000 stars up to magnitude 12 were investigated and about 7000 were considered members. 


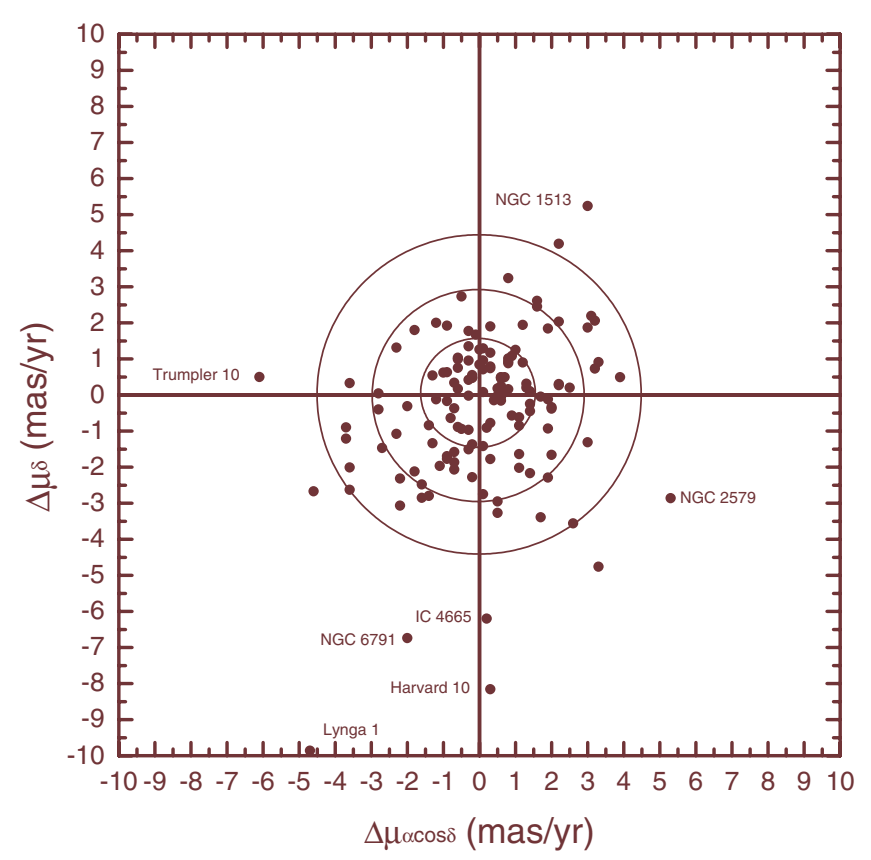

Fig. 2. Differences in mean proper motion (UCAC2 - Dias et al. 2001, 2002) in $\mu_{\alpha} \cos \delta$ and $\mu_{\delta}$. Circles show the regions of the one, two, and three standard deviation obtained in this study. The clusters outside of the circle of $3 \sigma$ are discussed in the text.

Considering the last comparison between our results and those from DLA, the differences follow a Gaussian distribution and, not surprisingly, $68 \%$ of the clusters are within $1 \sigma$ of the average difference. Figure 2 displays a diagram that simultaneously presents the differences in $\mu_{\alpha} \cos \delta$ and $\mu_{\delta}$ and shows more clearly this distribution. The remaining $11 \%$ farther points corresponding to 8 open clusters.

In the cases of the clusters Harvard 10, IC 4665, Ruprecht 147, and Trumpler 10, it seems that our analysis was unable to distinguish between members and background stars, probably because the clusters are large, nearby ones. Fortunately, good solutions were obtained by DLA for all these objects using the Tycho2 data.

However, for the open clusters NGC 1513, NGC 2579, and NGC 6791, we determined better mean proper motion estimates in this work, with intrinsically smaller dispersions and also larger numbers of member stars than in DLA.

\section{Summary}

All open clusters with known distances and $\delta \leq 52^{\circ}$ were investigated, and the mean absolute proper motions were obtained for 428 clusters, using kinematic data provided by the UCAC2 Catalogue and applying the statistical method of Zhao \& He (1990) for star membership determinations. For 75 open clusters, it is the first result, meaning an increase of $13 \%$ in the sample of open clusters with determined mean absolute proper motion.
In this work we have shown that the results generally agree with other cluster mean proper motion determinations as derived in the Hipparcos system. More particularly, our cluster proper motions agree well with the previous results from Dias et al. $(2001,2002)$ based on the Tycho2 Catalogue data, with the same typical dispersion of about $3 \mathrm{mas} / \mathrm{yr}$ in proper motions, but with a much larger number of identified astrometric member stars: about 42000 stars up to the 16th magnitude against about 7000 up to magnitude 12 with Tycho 2 .

Acknowledgements. We would like to acknowledge Dr. André Moitinho and Dr. Jacques Lépine for their comments. We wish to thank the anonymous referee for valuable suggestions to ward improving the paper. This project was supported by the FAPESP (grant number 03/12813-4). V. Flório acknowledges the FAPESP fellowship No.04/07919-0. This research made use of the Simbad data base, operated at the CDS, Strasbourg (France) and of the Digitized Sky Surveys produced at the Space Telescope Science Institute under US Government grant NAG W-2166.

\section{References}

Alessi, B. S., Moitinho, A., \& Dias, W. S. 2003, A\&AS, 410, 565

Baumgardt, H., Dettbarn, C., \& Wielen, R. 2000, A\&AS, 146, 251 (BDW)

Beshenov, G. V., \& Loktin, A. V. 2004, Astron. Astrophys. Trans., 23, $103(\mathrm{BL})$

Chen, L., Hou, J. 1., \& Wang J. J. 2003, AJ, 125, 1397

Clariá, J. J., Lapassate, E., Piatti, A. E., \& Ahumada, A. V. 2003, A\&A, 409, 541

Dias, W. S. 2000, Master Dissertation, IAG-USP, São Paulo, Brasil

Dias, W. S., \& Lépine, J. R. D. 2005, ApJ, 629, 825

Dias, W. S., Lépine, J. R. D., \& Alessi, B. S. 2001, A\&A, 376, 441 (DLA)

Dias, W. S., Alessi, B. S., Moitinho, A., \& Lépine, J. R. D. 2002, A\&A, 389, 871 (DAML02)

ESA 1997, The Hipparcos and TYCHO Catalogues, ESA SP-1200

Gulyaev, A. P., \& Nesterov, V. V., O Chetyrekhmillionnom kataloge zvezd (On the Four-Million Star Catalogue), Moscow: Izd. Mos. Gos. Univ., 1992

Høg, E., Fabricius, C., Makarov, V. V., et al. 2000, A\&A, 355, L27

Kharchenko, N. V., Pakulyak, L. K., \& Piskunov, A. E. 2003, Astronomy Reports, 47, 263 (KPP)

Loktin, A. V., Gerasimenko, T. P., \& Malysheva, L. K. 2001, Astron. Astrophys. Trans., 20, 607

Russeil, D. 2003, A\&A, 397, 133

Sanders, W. L. 1971, A\&A, 14, 226

Slovak, M. H. 1977, AJ, 82, 818

Urban, S. E., Corbin, T. E., Wycoff, G. L., et al. 1998, AJ, 115, 121

Vasilevskis, S., \& Rach, R. A. 1957, AJ, 62, 175

Zacharias, N., Urban, S. E., Zacharias, M. I., et al. 2004, AJ, 127, 3043

Zhao, J. L., \& He, Y. P. 1990, A\&A, 237, 54

Zhao, J. L., Tian, K. P., Xu, Z. H., \& Yin, M. G. 1982, Chin. Astron. Astrophys., 6, 293 